\title{
CAPACIDAD EMPRESARIAL, RESPONSABILIDAD SOCIAL $Y$ DESARROLLO NACIONAL
}

\author{
Robert Miranda Castillo
}

E-mail: d180028@unmsm.edu.pe

\section{PRESENTACIÓN}

El objeto del presente documento es examinar las caracteristicas y perspectivas de nuestro Perú desde la óptica de un desarrollo nacional, integral, sostenido y sustentable, y de la responsabilidad empresarial. La pregunta guía es: en qué medida la situación y las particularidades actuales del Perú, en el contexto internacional, permiten el desarrollo nacional, a partir de desplegar las capacidades emprendedoras. no sólo de los empresarios, sino también de los colaboradores (desde los gerentes hasta el último nivel jerárquico) y la responsabilidad social que debe asumir la clase dirigencial de nuestra patria.

El profesor de la Northern Illinois University, Luis Flores (1) señala que en el Perú "... nunca hemos sido buenos administradores. Tal vez hayamos sido buenos localmente, pero no en el ámbito mundial. ... porque hemos tomado como referencia a otras empresas del pais... Algo que me ha sorprendido es que empresas norteamericanas con menores recursos que ciertas empresas locales han logrado una posición dominante en el mercado mundial." Evidentemente necesitamos desarrollar tas competencias de calidad mundial de nuestra clase empresarial (incluidos gerentes y colaboradores)

En la revista Millonarios del mes de julio nos presentan un panorama sombrío desde el punto de vista de los inversionistas extranjeros, que debemos tomar en cuenta para desarrollar y diseñar el plan estratégico que necesita el Pais para los próximos 25 años y que a nuestro criterio debe ser liderado por la clase empresarial.

De hecho, es un reto ambicioso pretender que el sector empresarial peruano, asuma el papel determinante de liderar responsablemente el despliegue de capacidades de los más de veinticinco millones de peruanos, muchos de los cuales estamos șumidos en la desesperanza por no llegar a satisfacer, nuestras necesidades; mas esperamos la contribución y el interesante esfuerzo que el grupo de empresarios PERU 2021 y de otros esfuerzos que han realizado peruanos agrupados o de aportes individuales y que es urgente desarrollar e implementar concertadamente.

\section{NUESTRA REALIDAD}

Nuestro país y el mundo están inmersos en un vertiginoso proceso de cambios, pero estos excluyen prácticamente a la mitad de la población peruana y del mundo que no puedan beneficiarse mínimamente, con los resultados de la revolución del conocimiento (ver Mito del Desarrollo(2) y los resultados de la Cumbre Social 2000, Ginebra).

La tasa de crecimiento del producto en nuestro país (ver Cuadro $N^{\circ} 1$ ), en los últimos 20 años es menor a lo recomendado por los organismos internacionales. Para que un país pueda iniciar el despegue hacia el desarrollo social integral, sostenido y sustentable se necesitan tasas de crecimiento sostenido mayores al $6 \%$ y por lo menos durante veinte años.

En el cuadro $\mathrm{N}^{0} 1$, podemos observar cual ha sido el desempeño de nuestra economía en el contexto latinoamericano, a partir de 1980. Los tres períodos superiores al $6 \%$ (94.95 y 97) "no hacen verano" hecho que lastimosamente se confirma para 1999 con sólo $3 \%$ y para el presente año se estima sea alrededor al $4 \%$.

Una reflexión necesaria, aunque tal vez prematura a estas alturas, es preguntarse a dónde se destina la riqueza generada; parte de la respuesta nos la proporciona ef siguiente Cuadro $\mathrm{N}^{\circ} 2$ sobre el quantum de las importaciones de bienes y servicios, la cantidad de dichas importaciones que se destinan a tecnología. reestructuraciones, maquinaria y equipo y nos damos con la sorpresa que dichos montos son poco significativos en las inversiones y que la mayoria de la riqueza generada se destina a productos de consumo final del mercado doméstico, tenemos casos impresionantes del destino de la riqueza generada: por ejemplo, la adquisición de autos en el año 97 fue de aproximadamente 60 mil unidades (cifra nunca vista en nuestro país) entre nuevas unidades y usadas, pero para el año siguiente baja airededor del $50 \%$ y se estima que el año pasado sólo ingresaron una cuarta parte de dicha cantidad; hecho que nos demuestra la baja cultura de ahorro, a pesar de las altas tasas.

Mención aparte, merece el destino de las 
CRECIMIENTO DEL PRODUCTO INTERNO BRUTO

A PRECIOS CONSTANTES DE MERCAD

GROWTH OF GROSS DOMESTIC PRODUCT.

AT CONSTANT MARKET PRICE

[Tasas anuales medias / Average annual rates)

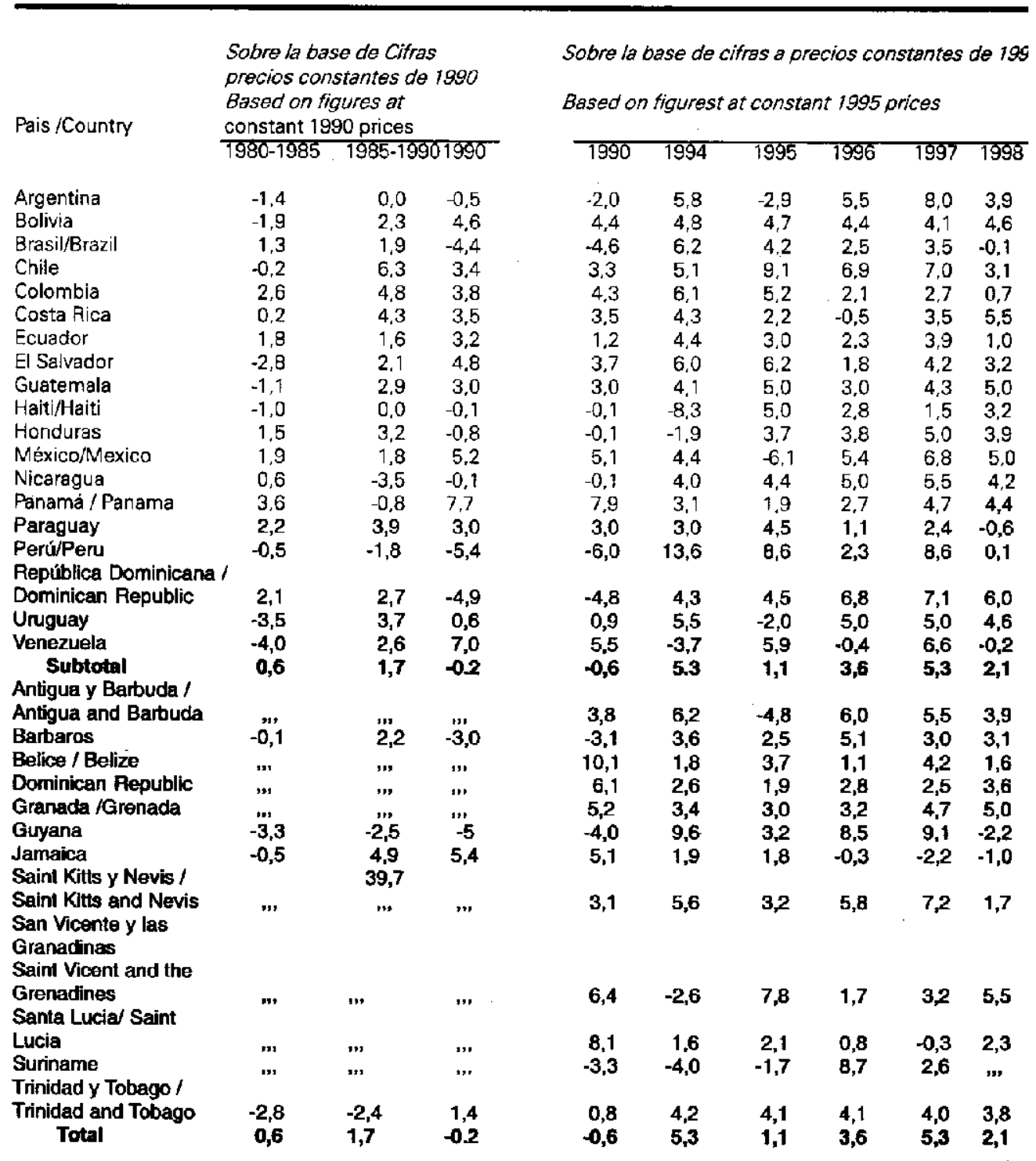

Fuente :CEPAL 1999. 
remesas de regalias y dividendos que obviamente se remiten a los paises de donde provienen las inversiones extranjeras, para los años 1996 y 97 significaron aproximadamente US\$6.2 millones y US\$25.0 millones, respectivamente\{3\}.

Hace más de dos años que hemos entrado en un franco proceso de recesión, que nos ha llevado a ubicarnos en el puesto número 86 del Desarrollo Humano(4), luego de Chile (puesto 31). Costa Rica (puesto 34). Argentina (36). Uruguay (38), doce paises más, por ejemplo Ecuador (puesto 73); sin embargo no debemos dejar de reconocer que estamos en la mitad de la lista de 174 paises (consuelo de tontos); en el puesto 80 en lo que se refiere al desarrollo por Genero y Familia (5).

Con el sinceramiento de la economía, las cifras preocupan, tanto por que indican que se han ocultado cifras, irresponsabilidad que debe ser sancionada ya que la realidad es otra; porque efectivamente $y$ luego del sinceramiento, la triste realidad: es que el PBI ha bajado a US\$ 2062,00 per cápita, como podemos observar en el Cuadro $\mathrm{N}^{\circ} 3$, y si miramos a nuestro vecino del sur Chile con más de US\$ 5000,00 per cápita. incluso nuestro vecino del norte nos supera tiene un per cápita mayor a los 2500,00 (6), quedamos a la zaga.

Las políticas de los últimos gobiernos, desde 1980 a la fecha y mucho antes también, no han orientado la economia al mercado internacional: seguimos pensando en el mercado interno, pequeño, poco atractivo. Tenemos una economía basada en las actividades primarias, muy poco se ha avanzado en la perspectiva de agregar valor a nuestros productos. observemos el Cuadro $\mathrm{N}^{\circ} 4$, para conocer, por ejemplo cual es la orientación de la economía chilena que tiene

CUADRO N० 02

CRECIMIENTO DEL QUANTUM DE LAS IMPORTACIONES DE BIENES Y SERVICIOS

GROWTH OF QUANTUM OFIMPORTS OF GOODS AND SERVICES

(Tasas anuales medias / Average annual rates )

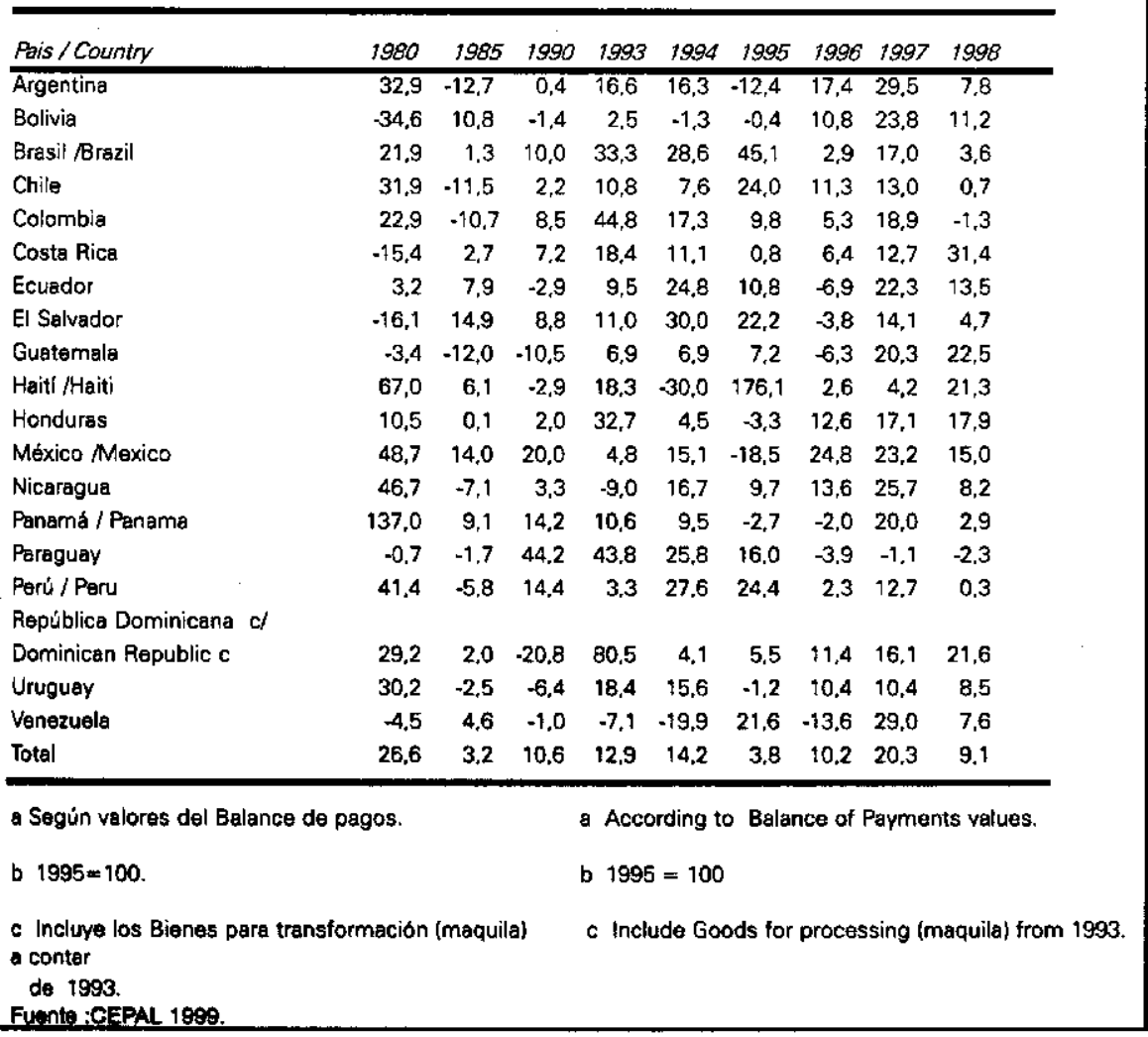


más del $33 \%$ de su economía orientada al mercado exterior, en tanto que la nuestra está alrededor de sólo el $13 \%$.

Nuestra Balanza Comercial indica, que el mayor componente está conformado por la exportación de materias primas tradicionales, teniendo en el cobre, harina de pescado, oro, entre otros pocos productos $y$ que las denominadas no tradicionales tienen una contribución mínima (ver Cuadros $N^{0} 5$ y $5 a$ ).

Evidentemente, hay muchos factores que deben concertarse para sentar las bases para que ef empresariado pueda aprovechar las ventajas comparativas de nuestro pais, la idea del presente artículo propone que el empresariado debe liderar ef

CUADRO N० 03

\begin{tabular}{|c|c|c|c|c|c|}
\hline \multicolumn{6}{|c|}{$\begin{array}{l}\text { PBI nominal y PBI per cápita } \\
\text { (Años base } 1979 \text { y } 1994 \text { ) }\end{array}$} \\
\hline An̄o & $\begin{array}{l}\text { Población } \\
\text { (miles) }\end{array}$ & \begin{tabular}{|l} 
PBI \\
Base \\
1979 \\
fUSS
\end{tabular} & $\begin{array}{l}\text { PBI } \\
\text { Base } \\
1994 \\
\text { nes) }\end{array}$ & $\begin{array}{l}\text { PBI } \\
\text { Per Cápita } \\
\text { (Base 1979) } \\
\text { (US\$) }\end{array}$ & $\begin{array}{l}\text { PBI } \\
\text { Per cápita } \\
\text { (Base 1994) } \\
\text { (US\$) }\end{array}$ \\
\hline 1994 & 23,130 & 49557 & 44808 & 2,142 & 1,937 \\
\hline 1995 & 23,532 & 58997 & 53653 & 2,507 & 2.280 \\
\hline 1996 & 23,947 & 60522 & 55827 & 2,527 & 2,331 \\
\hline 1997 & 24,371 & 64808 & 59056 & 2.659 & 2,423 \\
\hline 1998 & 24.801 & 62518 & 57005 & 2,520 & 2,298 \\
\hline 1999 & 25,232 & 57942 & 52028 & 2,296 & 2,062 \\
\hline \multicolumn{6}{|c|}{ Fuente: INEI } \\
\hline
\end{tabular}

gran movimiento por convertir a nuestro pais en competitivo, que convierta las inmensas ventajas comparativas en competitivas y que eso depende en gran medida de las decisiones de los líderes empresariales, políticos y todas las fuerzas sociales, entre las cuales deben estar las universidades.

Es necesario reconocer por parte de todos los ciudadanos peruanos, el empresariado peruana y los inversionistas internacionales que ha habido importantes avances en el escenario nacional al desarrollar un ambiente favorable para las inversiones. ambiente que se manifiestan en muchas esferas. algunas de las cuales mencionamos a continuación:

Pacificación del país.

Inserción de la economía en el proceso de globalización.

Programa de privatizaciones promotor de inversiones, aunque ha experimentado dificultades en los últimos años.

Marco legal adecuado para la inversión extranjera. Programa económico ceñido a las propuestas del Fondo Monetario Internacional, cuestionado por muchos sectores políticos, sociales del mundo y que están obligando, al parecer, a una profunda autoevaluación de su papel
Pero también es por todos conocido, que hay muchos factores negativos que han enrarecido el ambiente de las inversiones y que han repercutido en la salida de capitales de corto plazo, o han ahuyentado posibilidades de inversiones de largo plazo y han frenado el flujo de inversiones a nuestro país, entre estos factores tenemos:

Desequilibrio de la Balanza Comercial.

Deuda Externa.

Un proceso electoral plagado de dudas sobre su transparencia y legitimidad.

Imagen internacional deteriorada.

Sistema político tradicional.

Elevado indice de pobreza. CEPAL considera: "... que sólo basta un año de recesión para hacer a un lado cinco años de progreson (7).

Tasas de interés elevados.

Elevado índice riesgo-pais.

Seguimos siendo un país cuya estructura económica nos hace muy vulnerables ante el concierto mundial, no hemos podido desarrollar una industria y ofrecer servicios al mercado mundial, nuestra economia no está orientada al consumo externo, de alli que las 


\section{CUADRO $\mathrm{N}^{0} 04$}

\section{COEFICIENTES DE LAS EXPORTACIONES DE BIENES Y SERVICIOS}

\section{COEFICIENTS OF EXPORTS OF GOOOS AND SERVICES}

[Porcentajes del producto intema bruto a precios constantes /

Percentages of the gross domestic product at constant prices)

Sobre la base de cifras en

dólares a precios a 1990

Pats / Country Based on figures in dollars

at 1990 prices
Sobre la base de cifras en oblares a precios de 1995

Based an figures in dollars at 1995 prices

\begin{tabular}{|c|c|c|c|c|c|c|c|c|c|}
\hline & \multicolumn{3}{|c|}{ at 1990 prices } & \\
\hline & 1980 & 1985 & 1990 & 1990 & 1994 & 1995 & 1996 & 1997 & 1998 \\
\hline Argentina & 5,7 & 8.1 & 10.5 & 9.4 & 8.1 & 10.5 & 10.8 & 11.4 & 12.0 \\
\hline Bolivia & 14.5 & 13.5 & 18,7 & 19.2 & 21,6 & 22,6 & 22.5 & 20,8 & 20.4 \\
\hline Brasil / Brazil & 5,0 & 7.7 & 8,2 & 7.1 & 8,3 & 7.9 & 7.5 & 8.1 & 8.6 \\
\hline Chile & 24,3 & 26,8 & 33,6 & 26.6 & 28,6 & 29.7 & 31.9 & 32.7 & 33.8 \\
\hline Colombia & 17.4 & 15.1 & 21,6 & 11,6 & 12.3 & 13,1 & 13,4 & 13.9 & 15,0 \\
\hline Costa Rica & 23.5 & 26.9 & 34,4 & 33,6 & 40.8 & 42,7 & 47,4 & 47.7 & 51.1 \\
\hline Ecuador & 19.7 & 22.5 & 27,1 & 24,5 & 29.1 & 29.7 & 30,1 & 30,2 & 28,9 \\
\hline El Salvador & 27.8 & 23,7 & 20,3 & 16.8 & 20.2 & 21,6 & 23,1 & 26,6 & 26,3 \\
\hline Guatemala & 26,1 & 19.9 & 20,5 & 17.3 & 18,0 & 19,1 & 20,0 & 21,3 & 22.6 \\
\hline Haiti Maiti & 18,7 & 18.4 & 19,3 & 10.3 & 6,6 & 9,5 & 12,7 & 13,2 & 15.6 \\
\hline Honduras & 35,8 & 33.1 & 30.8 & 48,4 & 39,9 & 43,7 & 45,5 & 43.8 & 42.9 \\
\hline México Mexico & 10.3 & 15.3 & 18.6 & 14,8 & 19,7 & 24,6 & 27.9 & 30.5 & 32,1 \\
\hline Nicaragua & 20,1 & 14.7 & 18,3 & 27,5 & 32,8 & 34.2 & 44.7 & 44,1 & 39,6 \\
\hline Panamá /Panama & 90,6 & 82,6 & 83,5 & 108,6 & 93,7 & 100.9 & 92,1 & 97,0 & 89.1 \\
\hline Paraguay & 19.6 & 18,5 & 35.7 & 24.4 & 36.0 & 37.4 & 32.7 & 30,1 & 28,2 \\
\hline Perú Peru & 13.6 & 14,4 & 11,5 & 10.4 & 11,6 & 11.4 & 12,4 & 12,9 & 13,3 \\
\hline \multicolumn{10}{|l|}{ República } \\
\hline \multicolumn{10}{|l|}{ Dominicanal } \\
\hline \multicolumn{10}{|l|}{ Dominican } \\
\hline Republic & 24.8 & 23,3 & 28,2 & 20.0 & 46,9 & 49,0 & 49.5 & 53,4 & 54.7 \\
\hline Utuguay & 16.9 & 22.9 & 23,5 & 16.4 & 19,7 & 20.2 & 20.8 & 21.9 & 21.1 \\
\hline Venezuela & 27.6 & 30.8 & 38,7 & 27,2 & 28,9 & 29.3 & 31.8 & 32,3 & 33.0 \\
\hline Total & 10.3 & 12.9 & 15,3 & 12.4 & 14,5 & 15.9 & 16.8 & 18.0 & 18,9 \\
\hline
\end{tabular}

Fuente :CEPAL 1999.

inversiones se destinen a los sectores extractivos, como la mineria (Cuadro $N^{\circ} 6$ ) fundamentaimente $y$ en menor medida a otros sectores: ha habido inversiones en servicios, pero estos se han destinado a desarrollar el mercado interno (por ejemplo el mercado telefónico. finanzas, etcétera).

Está demostrado que aún no somos país atractivo para el capital internacional; al observar el flujo de las inversiones en el mundo, en 1998 la economia mundial registró un nuevo récard histórico para los ingresos de Inversión Extranjera Directa (IED), con más de 651440 millones de dólares, lo que supone unincremento de más de 38.5\% respecto al año anterior. incremento significativo, a pesar de la crisis mundial(8); el tema es que dichos IED, como se observa en el Figura $N^{\circ} 1$, se destinan a los mismos países desarrollados, sobre todo la Triada (Norteamérica, Unión Europea y Japón), es decir salen de un bolsillo para ir al otro bolsillo: 


\section{CUADRO NO5}

(Porcentajes del producto interno bruto a precios constantes )

Percentages of the gross domestic praduct at constant prices)

Sobre la base de cifras en

dólares a precios a 1990

Pais / Country Based on figures in dollars

at 1990 prices

$\begin{array}{lll}1980 & 1990\end{array}$

Sobre la base de cifras en

dólares a precios de 1995

Based on figures in dollars

at 1995 prices

\begin{tabular}{|c|c|c|c|c|c|c|}
\hline Argentina & 30,3 & 27,7 & 26,8 & 18,2 & 18.0 & \\
\hline Bolivia & 18,4 & 15,2 & 17.0 & 16.7 & 16.4 & \\
\hline Brasil & 27,2 & 24,8 & 22,8 & 21,6 & 21.6 & \\
\hline Chile & 19,3 & 18,2 & 18,5 & 20,0 & 19.6 & \\
\hline Colombia & 21,5 & $20 ; 0$ & 19.9 & 16,0 & 14.7 & \\
\hline Costa Rica & 19,5 & 19,5 & 19,4 & 18,4 & 18.9 & \\
\hline Ecuador & 20,0 & 19,8 & 15,6 & 20,9 & 22,3 & \\
\hline E! Salvador & 22.9 & 20.7 & 21.7 & 21,4 & 21,2 & \\
\hline Guatemala & $\uparrow 3,9$ & 13,2 & 12.5 & 12,5 & 11,9 & \\
\hline Haiti /Haiti & 18,2 & 16,2 & 15,8 & 15,8 & 7.2 & \\
\hline Honduras & .15 .4 & 15.7 & 16.3 & 17,1 & 17.1 & \\
\hline México & 18,6 & 18,0 & 19,0 & 19,4 & 18,8 & \\
\hline Nicaragua & 20.2 & 20.4 & 16,9 & 16,9 & 16,5 & \\
\hline Panamá & 10.1 & 9.4 & 9.5 & 8.6 & 8.9 & \\
\hline Paraguay & 18.8 & 17,8 & 17,3 & 16.9 & 15,9 & \\
\hline Perú & 29,3 & 27.1 & 27.3 & 23,2 & 24,3 & \\
\hline \multicolumn{6}{|l|}{ República } & \\
\hline Dominicana/ & 13.9 & 12,6 & 13,5 & 14,7 & 15,6 & \\
\hline Uruguay & 28,6 & 25,9 & 25.9 & 22.9 & 18,2 & \\
\hline Venezuela & 15,9 & 21.3 & 20,5 & 17,5 & 16,8 & \\
\hline Total & 24,3 & 22.7 & 21,8 & 19,9 & 19,6 & \\
\hline Antigua y & & & & & & \\
\hline Barbuda & & & $m$ & 3,1 & 2,3 & \\
\hline Barbados & 11,7 & 10.1 & 9.7 & 6.7 & 6,3 & \\
\hline Belice & $\ldots$ & ... & $\ldots$ & 13,8 & 13.5 & \\
\hline Dominica & $\ldots$ & $\cdots$ & $\cdots$ & 6,3 & 6,0 & \\
\hline Granada & $\ldots$ & $\ldots$ & ... & 6.1 & 6.3 & \\
\hline Guyana & 10,9 & 9.1 & 7.6 & 11,4 & 11.2 & \\
\hline Jamaica & 18,1 & 19,8 & 19,5 & 20.0 & 17,3 & \\
\hline \multicolumn{6}{|l|}{ Saint Kitts y } & \\
\hline Nevis & $\ldots$ & $\ldots$ & $\cdots$ & 12,3 & 10.6 & \\
\hline \multirow{2}{*}{\multicolumn{6}{|c|}{$\begin{array}{l}\text { San Vicente y } \\
\text { las Granadinas ... }\end{array}$}} & \\
\hline & $\ldots$ & ") & $m$ & 8,7 & 9,0 & \\
\hline Santa Lucia & $\ldots$ & $\ldots$ & $\ldots$ & 10.4 & 8.6 & \\
\hline Suriname & .. & .. & $\cdots$ & 19,5 & 15,9 & \\
\hline \multicolumn{6}{|l|}{ Trinidad y } & \\
\hline Tobago & 9,2 & 10,6 & 12,7 & 15,4 & 16.8 & \\
\hline Total & 24.1 & 22,6 & 21,8 & 19,9 & 19.5 & \\
\hline
\end{tabular}

Fuente :CEPAL 1999. 
PERU : EXPOATIACION DE LOS DIEZ PFODUCTOS PFINCIPALES

SEGLIN SU AARTCHFACION PORCENTUAL EN CADA ANOO

FERU: EX POATS OF THE TEN LEAOING PROOUCTS.

GY THEDR PER CENT AGE SHLARE FOR EACH YEAR

Productos prircipeles / Main products

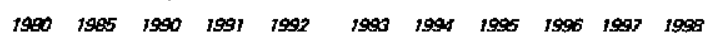

Oro (incluso oro platinedo) no monetario

en bruto semilabrado o en polvo

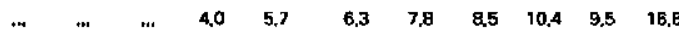

97101 Gold. nonrew or dust, noneurrent

$\begin{array}{llllllllllllll}\text { Cobre refinado ( incluido el relundido) } & 12.2 & 8.3 & 13,5 & 14.5 & 14.7 & 13.0 & 11.7 & 14,2 & 12,6 & 12,1 & 11.0 & 69212 \text { Copper refined }\end{array}$

Harina de carne lincluso el resiotuo de

grasasl y hering de pescado. impropios

para la elimentación humana.

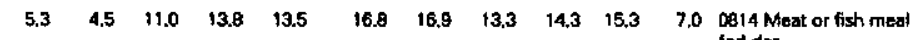

Mineral de zinc y sus concentrados

$\begin{array}{lllll}4.7 & 5.6 & 9.8 & 6.3 & 70\end{array}$

$\begin{array}{llll}5.3 & 4.8 & 4.3 & 4.7\end{array}$

fod der

Caf́t verde o lostado y sucedáneos

que contengan cald

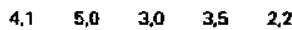

i.1 4,7

Rope inkevior de punto no elástica y sin cauchuter

Plata an bruto o semilabrada, can excepción da los chapadas. rossted.etc

Zin $y$ sus aleaciones, sin forjar
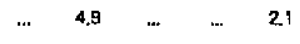

2.2

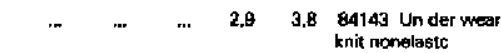

Mineral de plemo $\vee$ sus concentrados

Potróleos crudas

$$
\begin{array}{lllll}
8,1 & 6,8 & 4,5 & 3,7 & 3,0
\end{array}
$$$$
2,8
$$

$$
76,6 \quad 8,6
$$

Mineral de hisro y sus concentrados

[excepto piritas de hierro tostedas)

Mineral y concenirados de cobre

Productos derivadas del petróleo

Hilados e hilos de algodón gris (sin

blanquearl. to mercerizados. sin Bcort

dicionar para la venta al menor.

$$
\text { ... .. } 2,5
$$

$$
\begin{array}{lllll}
2,7 & 14,9 & 9,5 & 5,1 & 6,1
\end{array}
$$$$
\begin{array}{lllll}
6.0 & 4.0 & 2.4 & 2.5 & 2.4
\end{array}
$$

3.468111 Silwer unwinkd not nolld

Cobxe blis ter y demas cobre sin refinar

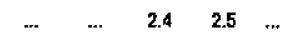

$\begin{array}{lllll}7.5 & 4.3 & 5.7 & 5.7 & 5.6\end{array}$

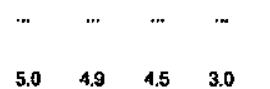

2. 6513 Grey cat ton yam in bulk

Articulas de joyarla de metales preciosos 2,6

...

... 68211 Coopper un refined

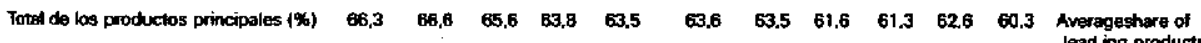
lead ing products $\left|x_{0}\right|$

Exportaciones al mundo

Exports to the wortd market

(millones de dólares\} 


\section{CUADRO $N^{\circ} 06$}

CRECIMIENTO DE LA PRODUCCION MINERA

GROWTHOF MINING PRODUCTION

(b) Excluíóo petróleo / Excluding petroleum

(Tasas anuales medias / Average annual rates)

\begin{tabular}{lllllllllll}
\hline Pais/Country & 1980 & $1985-$ & 1980 & 1992 & 1993 & 1994 & 1995 & 1996 & 1997 & 1998 \\
& 1985 & 1990 & 1990 & & & & & & &
\end{tabular}

\begin{tabular}{|c|c|c|c|c|c|c|c|c|c|c|c|}
\hline Argentina & 2.7 & 2.9 & 2.8 & $-11,0$ & $-41,8$ & $-12,2$ & 11,9 & $-2,3$ & 42.3 & 2,2 &. \\
\hline . & & & & & & & & 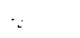 & & & \\
\hline Bolivia & $-9,5$ & 10.4 & 0,0 & 2.9 & 216.0 & 2.1 & 14,3 & $-6,6$ & 1.1 & $-1,4$ & \\
\hline Brasil /Brazil & 4,96 & 9.6 & 7,3 & -1.2 & $-10,6$ & 10,2 & 8,5 & 2.1 & 4,0 & 1.6 & \\
\hline Chile & 4.6 & 3,3 & 3,9 & 6,6 & 5,0 & 8.7 & 12,4 & 22.9 & 7,1 & 8,0 & \\
\hline Colombia & 20,9 & -0.8 & 9,5 & $-22,4$ & $-11,8$ & $-14,9$ & $-33,8$ & $-25,3$ & 4,8 & 5,0 & \\
\hline Cuba & $-2,5$ & 3.7 & 0.6 & $-4,2$ & -7.2 & 5,1 & 31.8 & 29.8 & 13.7 & 9.4 & \\
\hline Ecuador & 91.9 & -74.1 & 28.4 & $-89,1$ & -30.7 & 32.8 & 517.3 & $-2.7-$ & $-99.9-$ & $-100,0$ & \\
\hline Guyana & $-6,1$ & $-6,9$ & -6.5 & 94.0 & 62,3 & -7.5 & 2,8 & 28.4 & 7.1 & $-2,4$ & \\
\hline Honduras & 16.9 & $-12,3$ & 1,2 & -12.1 & $-30,3$ & $-2,4$ & 12,1 & 35,3 & 5,7 & $-7,0$ & \\
\hline Jamaica & $-12,2$ & 11,9 & $-0,9$ & $-2,2$ & $-1,6$ & 3.5 & -6.1 & 8.9 & 1.4 & 5.5 & \\
\hline México / Mexico & 3,1 & 3.7 & 3,4 & 0.4 & 9.2 & 4.2 & 8,7 & 2,5 & $-4,4$ & $-3,3$ & \\
\hline Nicaragua & $-16,4$ & 9,0 & $-4,5$ & 0.5 & 1.1 & $-8,0$ & 5.8 & 0,0 & 0 & 0,0 & \\
\hline Perú / Peru & 1,8 & -1.8 & 0 & $-3,9$ & 7,9 & 25,5 & 6.3 & 13.6 & 8.3 & 4,4 & \\
\hline \multicolumn{12}{|l|}{ República Dominicana } \\
\hline Dominican Republic & 1.4 & $-2,9$ & $-0,8$ & -9.6 & $-21,1$ & 25,1 & 1,4 & -3.2 & 8.7 & $-22,6$ & \\
\hline Suriname & $-5,3$ & $-2,7$ & -4.0 & 3.8 & $-1,8$ & 17.6 & $-4,5$ & 2.8 & 4.9 & 0.4 & \\
\hline Venezuela & 0.0 & 10.1 & 4.9 & -7.3 & 5,6 & 18.2 & 6,7 & -1.8 & 17,3 & $-22,0$ & \\
\hline Total & 3,2 & 4,6 & 10,1 & $-0,5$ & 0.0 & 9.9 & 8.7 & 11.0 & 5,3 & 3.8 & \\
\hline
\end{tabular}

Fuente :CEPAL 1999. 
FIGURA 1

\section{AMERICA LATINA Y EL CARIBE PARTICIPACIN EN LOS INGRESOS NETOS DE INVERSION EXTRANJERA, 1995 - 1998}

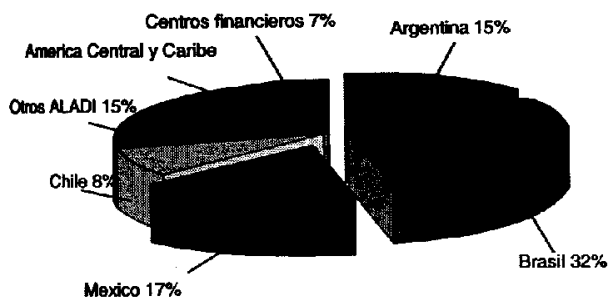

Empresariales de la División de Desarrollo Productivo y Empresarial, sobre la base de información proporcionada por el Fondo Monetario. Internacional. el Word mestment Report, 1999 de la Conferencia de las Naciones Unidas sobre Comercio

Yesarrollo (UNCTAD). Y los bancos centrales de los paises considerados.

Meditemos en las siguientes cifras, que nos hacen pensar cual es el destino y origen de la Inversión Extranjera Directa en el mundo y que tienen mucho que ver con el riesgo país que los inversionistas manejan para orientarlas (9):

El $93 \%$ de los ingresos de IED se orientan a los paises de la Triada, $y$

El $91 \%$ de las salidas de recursos financieros provienen de estos mismos países de la Triada (10).

Ahora detengámonos y observemos qué ocurre en América Latina y el Caribe, en esta parte del mundo ha registrado una tendencia diferente a la de los paises en desarrollo, y que indican una región que logró sortear una parte importante de las circunstancias negativas para atraer flujos financieros hacia nuestra región: convirtiéndonos en una alternativa nueva e interesante para los inversionistas internacionales, por ello la IED en Latinoamérica crece mientras que en el resto de países decrece la IED (11), como apreciamos en la Figura 2.

Pero el tema es, que algunos países latinoamericanos recibieron los mayores beneficios de IED, como se observa en el Figura $N^{\circ} 3$, siendo la "niña bonita" Brasil, pues en el periodo 1995-1999 participo con el $32 \%$, del total de IED que Latinoamérica recibió, en segundo lugar está México con $17 \%$, tercero Argentina con $15 \%$. Chile $8 \%$, Venezuela $6 \%$ y el Perú con menos del $1 \%$.

En el Cuadro N07, apreciamos el destino de la
FIGURA 2

\section{INGRESOS NETOS DE INVERSION EXTRANJERA DIRECTA EN REGIONES EN DESARROLLO, 1990- 1998}

(EN MILLONES DE DOLARES)

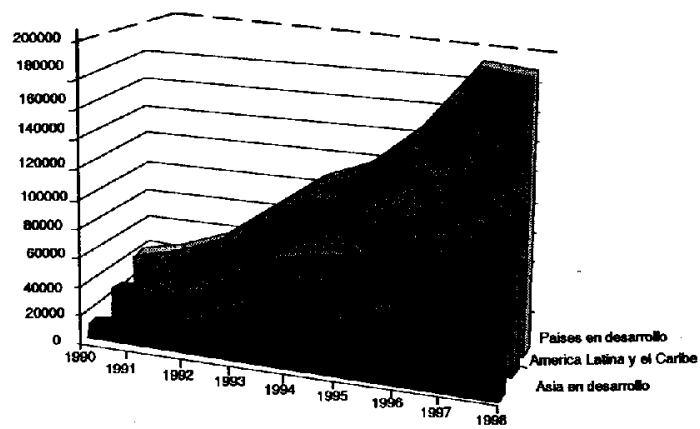

Fuenle: CEPAL, Centro de información de la Unidad de Inversiones y Estrategias Empresariales de la División de Desarollo Productivo y Empresarial, sobre la base de intormación proporcionada por ef Fondo Monetario Intemacional el Word investment Report 1999 de la conferencia de las Naciones Unidas sobre Comencio y Desartollo (UNCTAD), y los bencos centrales de los paises considerados.

\section{FIGURA 3}

\section{INGRESOS NETOS DE INVERSION EXTRANJERA DIRECTA}

(EN MILLONES DE DOLARES)

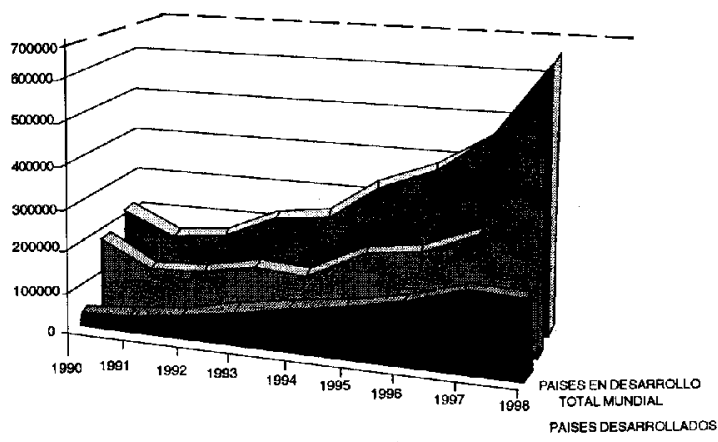

FUENTE: CEPAL, Centro de intomacion de la Unidad de Inversiones y Estrategias Empresariales de la Division de Desarrolio Productivo y Empresanial. Sobre la base de informacion proporcionada par el fondo Monetarlo Internacional y el World Invesiment Report, 1999, de la Conferencia de las Naciones Unidas sobre Comencio y Desarrollo (UNCTAD).

Los ingresos netos de IED comprenden todas aquellas entradas de capilal cuya finalidad es oner de la propiedad de una empresa local por arte de inversionistas extranjeros. El concepto de neto signitica que de los ingresos de capital se deducen las remesas de beneficios y las repatriaciones de capital. 


\begin{abstract}
PAISES DE LA ASOCIACION LATINOAMERICANA DE INTEGRACION (ALADI): INGRESOS NETOS DE INVERSION EXTRANJERA OIRECTA, 1990-1999
\end{abstract}

(En millones de dólares y porcentajes)

1990-1994

\begin{tabular}{|c|c|c|c|c|c|c|c|}
\hline \multicolumn{5}{|c|}{$1990-1994$} & \multicolumn{3}{|c|}{ PARTICIPACION } \\
\hline Paises & $\begin{array}{l}\text { (Promedio } \\
\text { anual) }\end{array}$ & 1995 & 1996 & 1997 & 1998 & $\begin{array}{l}1998 \\
\text { IPORC }\end{array}$ & 1999 a \\
\hline Argentina & 2971 & 5279 & 6513 & 8094 & 6150 & 9.5 & 21000 \\
\hline Bolivia & 85 & 393 & 474 & 731 & 872 & 1,4 & 800 \\
\hline Brasil & 1703 & 4859 & 11200 & 19650 & 31913 & 49.5 & 31000 \\
\hline Chile & 1219 & 2957 & 4637 & 5219 & 4638 & 7.2 & 8900 \\
\hline Colombia & 818 & 969 & 3123 & 5703 & 3038 & 4.7 & 350 \\
\hline Ecuador & 293 & 470 & 491 & 695 & 831 & 1,3 & 470 \\
\hline México & 5430 & 9526 & 9186 & 12831 & 10238 & 15,9 & 10000 \\
\hline Paraguay & 98 & 155 & 246 & 270 & 256 & 0.4 & 100 \\
\hline Perú & 785 & 2000 & 3226 & 1785 & 1930 & 3,0 & 1500 \\
\hline Uruguay & .. & 157 & 137 & 126 & 164 & 0,3 & 100 \\
\hline Venezuela & 836 & 985 & 2183 & 5536 & 4435 & 6.9 & 1200,0 \\
\hline Total & 14238 & 27750 & 41416 & 60640 & 64465 & 100,0 & 75420 \\
\hline
\end{tabular}

Fuente : CEPAL Centro de Información do la Unidad de Inversiones y Estrategias Empresariales de la División de Dessarrollo Productivo y Empresarial, sobre la base de información proporcionada por el Fondo Monetario Internacional. el Wort Investment Report. 1999. de la Conferencia de las Naciones Unidas sobre Comercio y Desarrollo (UNCTAD). y los bancos centrales de cada pais.

Estimaciones de la Unidad de Inversiones y Estrategias Empresariales de la CEPAL sobre la base de provecciones de los bancos centrales de cada pais, 22 de noviembre de 1999.

Inversión Directa Extranjera en los países miembros de la Asociación Latinoamericana de Integración (ALADI). en 1998 ocupamos el $8^{\circ}$ puesto de once paises. con una participación de sólo el $3 \%$. el gigante Brasil recibe un impresionante $49.5 \%$, luego México con $15.9 \%$ pues los efectos del TLCNA del año 94 al parecer ya cumplieron los objetivos para las transnacionales norteamericanas y dejan México; Argentina $9.5 \%$ y Chile $7.2 \%$ entre los destinos más importantes para los inversionistas

La captación de IED para los paises de la ALADI aumentan de manera sostenida. hecho como hemos indicado no es la tendencia registrada en los otros paises en desarrollo del mundo, pero que sí está presente en Latinoarnérica una región interesante para los inversionistas internacionales; en 1998 crece algo más de $10.6 \%$ respecto al año anterior, pero esta tendencia no es favorable para nuestro país.

Mención aparte merece dos paises: Brasil y
México. El primero capta el $42 \%$ de la IED en 1998. de total de paises latinoamericanos, a pesar de la situación inestabilidad de su economía desde 1997 (12), pero debido a su programa de privatizaciones y a la progresiva y rápida desrregulación de algunos sectores claves de su economía. van permitiendo una masiva llegada de inversionistas extranjeros a las áreas de servicios e industria de la economia brasileña (13).

En el período 1990-1994 fue México ef pais que recibió el mayor parcentaje de IED, casi un tercio del total destinado para ALADi por ser el único país en que las empresas transnacionales, mayormente estadounidenses, buscan eficiencia en México y establecieron plantas para aprovechar los salarios más bajos y la política mexicana de facilitar operaciones combinada con ventajas arancelarias que otorga ef mecanismo de producción compartida de los EEUU, así como también de las consecuencias del Tratado de Libre Comercio entre Méxica. Canadá y Estados Unidos, que entró en vigencia en 1994 (14). 
Un pais que también merece nuestra atención as Chile, con menores ventajas comparativas al nuestro, se ha convertido en el pais con ventajas competitivas atractivas para los inversionistas internacionales y con una gran capacidad de captar mayores inversiones, ha crecido sostenidamente desde 1990 (ver Cuadro Nº). podemos referir que en el año 98 . Chile ha recibido más de un millón ochocientos mil turistas extranjeros, que para nuestro Gobierno el objetivo son un millón de turistas para el presente año 2000, ni que hablar de la pequeña isla del Caribe: Cuba, con más de un millón y medio de turistas.

El día 17 de julio del presente, El Comercio, en su primera página, informa que Inglaterra está criando alpacas para producir tela, un ejemplo claro de cómo perdemos ventajas comparativas y que las estrategias del Estado y Gobierno peruanos son insuficientes para revertir ına tendencia de muchas décadas en casi todos los sectores.

La apertura, destregulación, fusiones $y$ adquisiciones (bajo formas de privatizaciones $y$ concesiones) han sido las oporturidades que han aprovechado los inversionistas internacionales para decidir en qué país invertir, mediante la modalidad de Inversión Extranjera Directa (AED), en América Latina. sobre todo en el MERCOSUR, México y Chile.

Las compañías estadounidenses mantienen su predominio en términos del valor de los activos y ventas en Latinoamérica, aunque en los últimos años algunas empresas europeas inician una fuerte presencia en la región. En términos de sectores las empresas europeas se orientan a los mercados de servicios (caso de telefonía en Perú. Chile, Argentina) y las estadounidenses a la industria (industria automovilística en Brasil, Argentina, etc.).

Respecto a la educación, prioridad número uno de los estados que han implementado estrategias de desarrolio con resultados favorables, tales como Japón, Chile, y en general todos los países desarrollados, a continuación tenemos el caso de Chile (15):

\begin{tabular}{llr} 
& PERU & \multicolumn{1}{l}{ CHILE } \\
$\begin{array}{llr}\text { Sueldo promedio } \\
\text { profesores }\end{array}$ & 600 soles & 800 dólares \\
$\begin{array}{l}\text { Inversión por } \\
\text { alumno }\end{array}$ & $\mathbf{1 6 2}$ dólares & 1,800 dólares
\end{tabular}

El concepto de responsabilidad social de la empresa ha evolucionado, desde la propuesta filantrópica de Andrew Carnegie (16), pasando por Milton Friedman: "... en la empresa sólo existe una responsabilidad social: utilizar sus recursos en actividades encaminadas a incrementar sus utilidades, mientras respete las reglas del juego"(17), a la posibilidad de aceptar que la responsabilidad social corporativa de la empresa (RSC) sea la "... aplicación forzada de ciertos tipos de comportamiento a partir de fuentes externas... para responder a necesidades sociales"(18), así en la responsabilidad por el ambiente interno, hay una interrelación entre empresa y gobierno por desarrollar ciertas prácticas respecto a terceros. tales como clientes, competencia, gobierno, empleados. proveedores y acreedores 19 \}, que están clasificadas en responsabilidades: discrecionales, éticas, legales $y$ económicas

Por tanto la responsabilidad social corporativa de las empresas, mejor dicho de los que dirigen las organizaciones involucra a factores internos y externos. entre las que tenemos responsabilidad sobre sus colaboradores, accionistas, clientes, proveedores, competidores. gobierno, medio ambiente, etc., por lo tanto debe configurar sus actividades en una pe-pectiva de desarrollo integral, sostenido $y$ suistentable de la misma humanidad.

Por nuestra parte agregamos, que no hay otra perspectiva si queremos asumir los conceptos y técnicas de calidad total, excelencia y además que dichas organizaciones deben administrase bajo los criterios de gerencia estratégica, teniendo en cuenta que el concepto básico de estrategia es conocer al competidor para diseñar una estrategia de ataque; sea frontal, defensiva, posicionamiento, etc., en esa perspectiva cabe pensar en el empleo, tratamiento de los residuos, la capa de ozono, la cultura proactiva, la democracia, el sistema politico, la cultura proactiva de tributación, distribución de la riqueza، etcétera, etcétera. que nos obliga referir al bate peruano "...hay hermanos muchisimo que hacer".

Un tratamiento especial merece el tema de la pobreza que cubre a más del $40 \%$ de la población peruana, los avances logrados en algunos años de la

En el último período del presente gobierno. hemos retrocedido en el proceso de integración social. tanto así, que tenemos un Perú cada vez más dividido. altamente centralizado en los aspectos económicos (Lima concentra la producción, finanzas, transacciones económicas, poder político, legislativo. la riqueza; $y$ yendo, incluso, contra la corriente mundial que propugna la descentralización. la no violencia. autonomía, equidad, formalización, integración social, otorgamiento de poderes, etc.). políticos, culturales y tecnológicos.

\section{PROPUESTA}

En estas circunstancias y dadas las perspectivas proponemos:

1. Concertar esfuerzos entre los sectores económicos, políticos, sociales, educativos, Estado y Gobierno para formular e implementar un Plan Estratégico de Desarrollo, Integral, Sustentables y Sostenido. pasando por renegociar, con los organismos financieros y de desarrollo internacionales.

2. Promover en todos los niveles del emprèsariado 


\section{CUADRO N0 08}

CONSUMO DE ENERGIA ELECTRICA POR HABITANTE

PER CAPITA CONSUMPTION OF ELECTRIC ENERGY

(Kilowatt horas $(\mathrm{kWh}) / \mathrm{Kil}$ owatt hours (kwh))

\begin{tabular}{|c|c|c|c|c|c|c|c|c|c|}
\hline Pais / Country & 1980 & 1985 & 1990 & 1991 & 1992 & 1993 & 1994 & 1995 & 7996 \\
\hline \multicolumn{10}{|l|}{ Antigua Y Barbuda / } \\
\hline Antigua and Barbuda & 760 & 939 & 1484 & 1484 & 1462 & 1462 & 1470 & 1485 & 1485 \\
\hline Argentina & 1406 & 1492 & 1591 & 1666 & 1761 & 1880 & 1957 & 1993 & 2076 \\
\hline Bahamas & 4024 & 3514 & 3725 & 3697 & 3625 & 4409 & 4516 & 4512 & 4558 \\
\hline Barbados & 1307 & 1535 & 1821 & 2043 & 2065 & $211 \uparrow$ & 2194 & 2333 & 2453 \\
\hline Belice /Belize & 372 & 433 & 561 & 547 & 711 & 876 & 803 & 808 & 808 \\
\hline Bolinia & 281 & 237 & 326 & 339 & 352 & 348 & 400 & 409 & 425 \\
\hline Brasil/Brazil & 1148 & 1442 & 1673 & 1738 & 1740 & 1824 & 1855 & 1948 & 2016 \\
\hline Chile & 1058 & 1158 & 1402 & 1499 & 1651 & 1743 & 1804 & 2105 & 2169 \\
\hline Colombia & 890 & 1054 & 1020 & 1028 & 999 & 1095 & 1150 & 1184 & 1140 \\
\hline Costa Rica & 977 & 1047 & 1216 & 1218 & 1257 & 1308 & 1353 & 1370 & 1369 \\
\hline Cuba & 1027 & 1208 & 1381 & 1190 & 1032 & 973 & 1071 & 1136 & 1201 \\
\hline Dominica & 151 & 286 & 423 & 437 & 437 & 437 & 479 & 521 & 521 \\
\hline Ecuador & 420 & 512 & 616 & 663 & 667 & 678 & 727 & 729 & 792 \\
\hline El Salvador & 322 & 374 & 450 & 455 & 472 & 536 & 575 & 593 & 600 \\
\hline Granada /Grenada & 234 & 258 & 604 & 659 & 692 & 707 & 761 & 957 & 1033 \\
\hline Gıatemala & 223 & 220 & 266 & 278 & 304 & 327 & 325 & 355 & 342 \\
\hline Guyana & 484 & 409 & 392 & 316 & 310,0 & 310 & 309 & 402 & 408 \\
\hline Haiti /Haiti & 54 & 69 & 68 & 66 & 60 & 53 & 41 & 69 & 82 \\
\hline Honduras & 250 & 281 & 407 & 422 & 447 & 469 & 489 & 486 & 485 \\
\hline Jamaica & 1012 & 978 & 1038 & 890 & 914 & 1561 & 1948 & 2367 & 2420 \\
\hline México / Mexico & 972 & 1176 & 1455 & 1479 & 1494 & 1522 & 1621 & 1665 & 1754 \\
\hline Nicaragua & 380 & 381 & 382 & 388 & 398 & 391 & 388 & 403 & 423 \\
\hline Panamá / Panamá & 1001 & 1123 & 1203 & 1248 & 1269 & 1330 & 1376 & 1371 & 1486 \\
\hline Paraguay & 213 & 328 & 575,0 & 597 & 566 & 728 & 812 & 913 & 1601 \\
\hline Perú /Peru & 567 & 615 & 641 & 660 & 588 & 646 & 677 & 741 & 837 \\
\hline \multicolumn{10}{|l|}{ Republica Dominicana/ } \\
\hline Dominican Pepublic & 597 & 659 & 520 & 537 & 754 & 779 & 805 & 832 & 860 \\
\hline \multicolumn{10}{|l|}{ Saint Kitts y Nevis / } \\
\hline Saint Kitts and Nevis & $\cdot$ & 745 & 881 & 2000 & 2000 & 1683 & 1805 & 1976 & 2000 \\
\hline Santa Lucia/Saint Lucia & 483 & 566 & 782 & 778 & 781 & 775 & 800 & 796 & 799 \\
\hline \multicolumn{10}{|c|}{ San Vicente y las Granadinas } \\
\hline \multicolumn{2}{|c|}{ Saint Vicent and the Grenadines 273} & 286 & 467 & 491 & 523 & 564 & 577 & 580 & 584 \\
\hline Suriname & 4480 & 3457 & 3741 & 4188 & 3884 & 3911 & 3934 & 3946 & 3944 \\
\hline \multicolumn{10}{|l|}{ Trinidad y Tobago / } \\
\hline Trinidad and Tabago & 1904 & 2545 & 2945 & 3039 & 3222 & 3147 & 3247 & 3413 & 3576 \\
\hline Uruguay & 1182 & 1302 & 1580 & 1671 & 1751 & 1808 & 1864 & 1950 & 2017 \\
\hline Venezuela & 2298 & 2771 & 3051 & 3006 & 3215 & 3400 & 3414 & 3422 & 3353 \\
\hline TOTAL & 1049 & 1262 & 1393 & 1425 & 1445 & 1517 & 1568 & 1633 & 1691 \\
\hline
\end{tabular}


nacional y de los colaboradores un compromiso por convertirse en el pilar del Plan del Desarrollo Nacional Integral, Sostenido y Sustentable, en el que participen todos los sectores de la Sociedad y el Estado. convirtiendo a este último en un promotor eficiente del desarrollo, teniendo al mundo como nuestro mercado.

3. Identificar los sectores en donde el Perú tiene ventajas comparativas y competitivas para que sea el pilar del Plan de Desarrollo Nacional.

4. Convertir a las universidades y centros tecnológicos en el sustento de adaptación, rescate y generación de las ciencias, tecnología y arte.

5. Promover el cambio de cultura de paz, a partir de valores y actitudes proactivas, sistema educativo de calidad internacional, sobre todo en las nuevas generaciones.

6. Desarrollo de un sistema político plural y participativo, capaz de llegar a acuerdos, procesar diferencias $y$

1 El Comercio, 4 de enero del 2000.

2 OSWALDO DE RIVERO. El mito del desarrollo, Lima 1998.

3 CONITE, 1996, referencia COEM '96. ESAN.

4 www.undp.org/

5 www.undp.org/hdro/98hdi.htm

6 www.undp,org/hdro/98hdi.htm

7 Revista,Business, junio 2000.

B FMI, 1999; Instituto de Finanzas Internacionales, 1999:UNCTAD, 1999.

9 UNCTAD. 1999a، p.45.

10 www.eclac.cl/

11CEPAL. la inversión extranjera en América Latina y el

Caribe, 1999.

12 CEPAL, 1999b. gestar acuerdos nacionales en torno al desarrollo integral, sostenido $y$ sustentable.

7. Reducción de la pobreza y mejoramiento de los sistemas de redistribución de la riqueza. movilidad social, programas sociales agresivos de cobertura total, sobre todo en los jóvenes y mujeres de los sectores más vulnerables.

8. Promover la eficiencia y eficacia bajo criterios de competitividad, cambio y globalización.

9. Un poder judicial justo y equitativo, que siente las bases de un enfoque integrado de politicas económicas y sociales para fomentar la competitividad, la solidaridad, la cohesión social y el desarrollo humanos integral, sustentable $y$ sostenido.

El reto es impresionante pero fascinante para empresarios, gerentes, profesionales, docentes en administración, trabajadores y organizaciones vinculadas al quehacer empresarial.

13 www.eclac.cl/

14 www.eclac.cl/espanol/Publicaciones/inverg9/ entrevista.htm

15 Diario, La República, 10 de julio del 2000.

16 STONER. JANES Y FREEMAN, R. EDWARD.

Administración. Edi. Prentice Hall International. México. 1992.

17 Friedman, Milton. Capitalismo and Freedmon. 1963.

18 Kast, Fremont y Rosenzwig. Administración de las organizaciones. Edi. Mc Graw Hill. México. 1994

19 Ivancevich y otros. Gestión, Calidad y Competencia. Edi. Irwin. Colombia. 1996.

(t) Licenciado en Administración - Universidad Nacional de Trujillo.

Magister en Administración de Empresas y Negocios de la Universiadad de los Lagos Chile y Universidad de San Pedro Chimbote.

Oocente Universitario Asesor y Consuttor de Empresas.

Miembro de la Unidad de investigación de la Facultad de

Ciencias Administrativas - UNMSM 